\title{
Shielding effectiveness assessement of a coaxial cable design with a combination of numerical and analytical solutions
}

\author{
Clement Pornin*, Tan Phu Vuong*, Pascal Xavier*, Gilbert Angenieux* \\ * Univ. Grenoble Alpes, Univ. Savoie Mont Blanc, CNRS, Grenoble INP, IMEP-LAHC, 38000 Grenoble, France , \\ \{clement.pornin, tan-phu.vuong, pascal.xavier1\}@grenoble-inp.fr, gilbert.angenieux@univ-smb.fr
}

\begin{abstract}
This paper introduces a novel approach to assess the shielding effectiveness of coaxial cables design by combining the numerical electromagnetic modeling and an analytical solution. A simplified numerical 3D model is built with the software CST microwaves. The 3D model is limited by the complexity of the braided structure of the coaxial cable's shield. Hence, in this model, the woven structure of the braided shield is simplified by using nonwoven crossed strands. The effect of the weaving is added separately using an analytical solution. A comparison between measurement results extracted from literature, raw results of simulation and simulation results corrected with analytical formula is given. Finally the relevance of the presented method is discussed.
\end{abstract}

Index Terms-Coaxial cable, EMC, Transfer impedance, shielding effectiveness, numerical modeling

\section{INTRODUCTION}

Development of the industry and telecommunication, in particularly since the past century, rises ever more the density of integration of electronic devices. Insuring signal integrity through transmission lines connecting all these devices becomes a very sensitive task. Electromagnetic compatibility has to be considered at all the steps of design of a device. Coaxial transmission lines are widely used since the beginning of the $X X^{t h}$ century in order to insure the signal integrity of transmitted data. The first coaxial line had solid copper shield, which gave very good shielding properties but wasn't flexible. For the sake of the flexibility and the feasibility of the installation process, the braided shield is used in most cases. Nevertheless, the shielding properties of the braided metal is not as good as solid tubes and the assessment of a new design is hard to process. Many researchers tried to define analytical solutions in order to directly obtain the shielding properties of a cable from the building parameters of the braided shield. The most interesting papers in this field will be slightly reviewed in the first section. Each of these works had brought useful elements on the way to the exact theory to predict the behavior of the braided shields against external spurious signals but always kept some discrepancies with the reality of the measurement. On the other hand, numerical electromagnetic modeling of coaxial cables have been more recently introduced by fewer authors and appears to be promising. Some of these works will be slightly reviewed in the second section.

Organization of the paper: This paper has four parts.

- The first part is a brief review of previous works on the theory of the coaxial cable shields.

- The second part introduces some numerical electromagnetic models built by other authors.

- The third part gives our numerical model and and its peering with analytical solutions.

- The fourth part contains the results obtained, compared with other measurement and theory and gives the conclusions.

\section{THEORIES ON COAXIAL CABLE SHIELDS}

\section{A. The first approach brought by Schelkunoff}

The first research on coaxial transmission lines shielding effectiveness was brought by Schelkunoff [1] in 1934. His work was focused on coaxial transmission lines with a solid copper tube as a shield. These transmission lines where used in radar installations. He gave a lot of theoretical analysis to understand the behavior of these cables against ElectroMagnetic Interferences (EMI). He defined the notion of transfer impedance $\left(Z_{t}\left[\Omega \cdot m^{-1}\right]\right)$, which is intrinsic to the cable itself. This parameter is calculated using equation(1), with reference to the measurement setup depicted in figure 1 .

$$
Z_{t}=\frac{\Delta V}{I_{s} \times L}\left[\Omega \cdot m^{-1}\right]
$$

Where $\Delta V[\mathrm{~V}]$ is the potential difference between the shield and the cable core appearing at one end of the cable when a current $\mathrm{I}_{\mathrm{S}}[\mathrm{A}]$ flows on the shield of the cable under test (CUT). L [m] is the length of the sample of cable. One end of the cable is in short circuit and the other end is connected to the high input impedance of the tension measurement device.

The transfer impedance parameter illustrates the coupling between the external magnetic wave associated to the shield current $\mathrm{I}_{\mathrm{s}}$ with the transverse electromagnetic (TEM) wave propagating inside the cable. The purpose of the shield is to obtain the lowest value of transfer impedance, in order to minimize this coupling. 


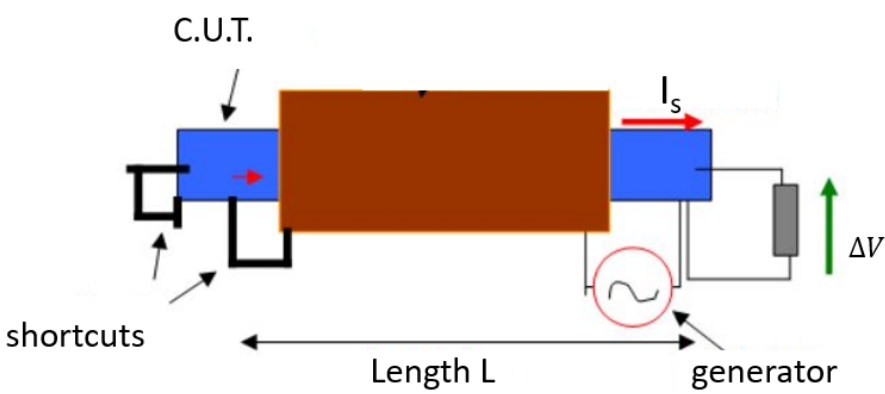

Fig. 1. Schematic representation of the triaxial setup for measurement of transfer impedance

\section{B. Theorical studies on the braided shields}

Vance [2] used the theory brought by Kaden [3] and Marcuvitz [4] , who studied the leakage of electromagnetic waves through perforated metallic tubes. His purpose was to model the coupling through holes appearing in the braid as shown in figure 2. Vance said that the magnetic coupling through the holes, as shown in figure 3 , can be modeled as an inductive term $\mathrm{M}_{12}$ in the expression of $\mathrm{Z}_{\mathrm{t}}$ of the equation 5 . The inductive coupling through hole is socalled the hole inductance. Other authors made improvements of Vance's model. For exemple Kley's semi empirical description in [5] and Sali [6]. For the sake of simplicity, we here took as a reference the recently published paper [7] describing the behaviour of braided shield against EMI. This paper presents a reliable review of existing models in order to enable the reader to choose the most relevant model for the considered cable.

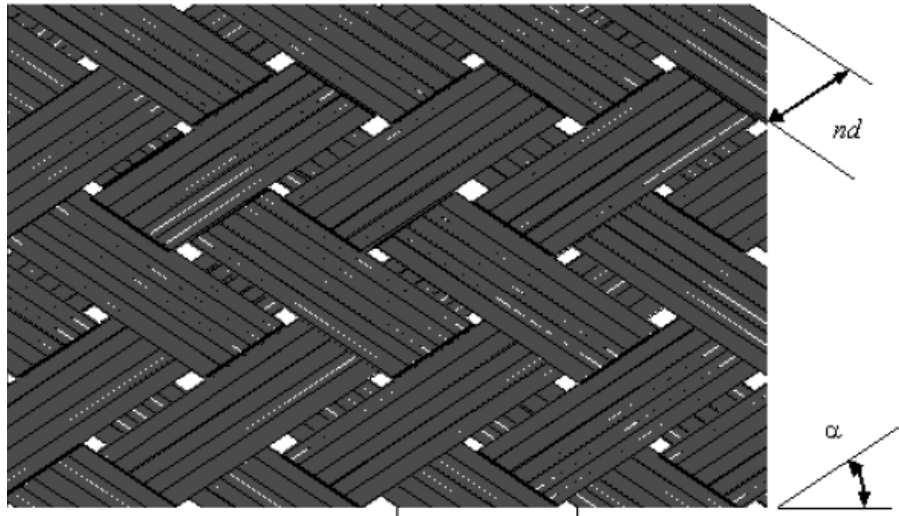

Fig. 2. representation of the braided structure with main building parameters [8]

The building parameters of the braid are :

- $\mathrm{n}$ number of strands in a spindle

- $\zeta$ the number of spindles

- $d$ the diameter of elementary conductors of the braid [m]

- D the diameter of the shield

- $\sigma$ the conductivity of the braid's metal [S.m ${ }^{-1}$ ]

- $\alpha$ the angle between braid conductors and the axis of the cable - the angle between two crossing strands is $2 \alpha$
In [2], Vance linked these primary parameters with secondary parameters: the dimensionless filling factor $\mathrm{F}$ (equation 2 ), the number of carrier crossings and holes per unit length, respectively $\mathrm{P}$ (equation 3 ) and $\nu$ (equation 4 ).

$$
\begin{gathered}
F=\frac{n \zeta d}{4 \pi D \cos \alpha}[\phi] \\
P=\frac{\zeta \sin \alpha}{4 \pi D \cos \alpha}=\frac{\zeta}{4 \pi D} \tan \alpha\left[\mathrm{m}^{-1}\right] \\
\nu=P \zeta=\frac{4 \pi D \sin \alpha \cos \alpha}{n^{2} d^{2}} F^{2}\left[\mathrm{~m}^{-1}\right]
\end{gathered}
$$

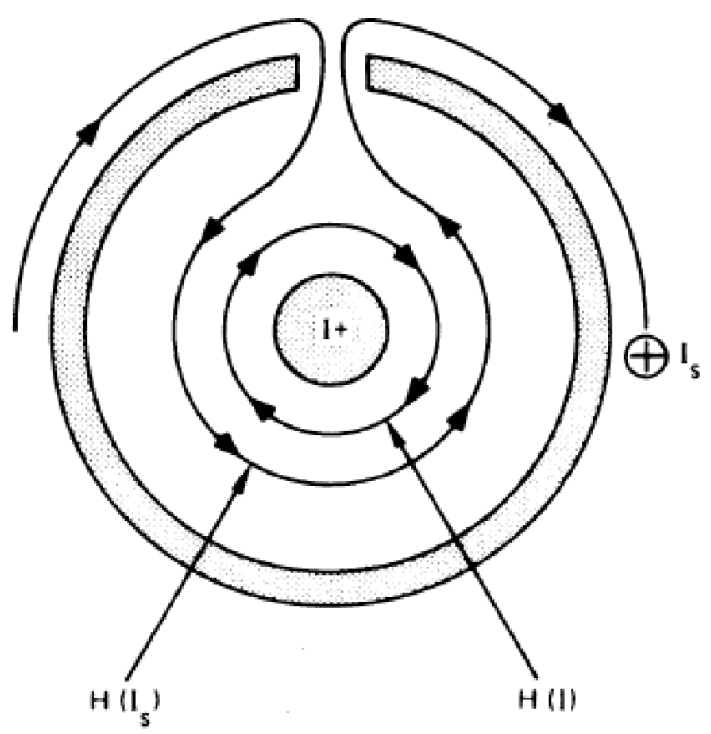

Fig. 3. Magnetic coupling through braid's hole [2]

$$
Z_{t}=R_{0} \times \frac{\gamma \partial}{\operatorname{sh}(\gamma \partial)}+j \omega M_{12}\left[\Omega . m^{-1}\right]
$$

Where $\mathrm{R}_{0}[\Omega]$ is the DC resistance of the braid calculated with equation 6 and $\partial=\mathrm{d} / \delta$; where $\delta$ is the skin depth defined by equation 7 . The term $\frac{\gamma \partial}{s h(\gamma \partial)}$ corresponds to the diffusion of electromagnetic field through the thickness of the metal braid. The diffusion term dominates the transfer impedance at low frequency. $\gamma=1+j$ is the complex propagation constant in the shield according to [9].

$$
\begin{gathered}
R_{0}=\frac{4}{n \zeta \sigma \cos \alpha d^{2}}\left[\Omega \cdot m^{-1}\right] \\
\delta=\frac{1}{\sqrt{\pi f \mu \sigma}}[m]
\end{gathered}
$$

Where $\mu$ is the magnetic permeability of the braid metal $\left[\mathrm{H}_{\mathrm{m}} \mathrm{m}^{-1}\right]$ and $f[\mathrm{~Hz}]$ the considered frequency.

The mutual inductance term of Vance's model in [2]is only based on the hole inductance and is defined by equation 8 .

$$
M_{12_{\text {Vance }}}=\nu \frac{\mu_{0} m}{4 \pi^{2} D^{2}}[H]
$$


Where $\mathrm{m}$ is the magnetic polarizability of the rhombic shape of the hole given in an improved form in [10] [11]. In [12], the authors widely reviewed the notion of hole inductance and gave the equation 9 to approximate $\mathrm{m}$.

$$
m=\frac{2}{3 \pi}(2-\cos \alpha) \sqrt{\tan \alpha}
$$

The most reliable form of the hole inductance $M_{h}$ is available in [7] but we do not detail this model here for the sake of brevity.

Tyni [13] said that the magnetic fields respectively corresponding to crossing spindles interract. Hence, he added the braid inductance term $\left(M_{b}\right)$ to the Vance's analytical formula to consider this phenomena. The braid inductance is defined by equation 10 .

$$
M_{b}=-\mu \frac{d}{4 \pi D} \frac{\hat{h}}{d}\left(1-\tan ^{2} \alpha\right)
$$

Where $\hat{h}[\mathrm{~m}]$ is the average height of the loops formed by the crossing conductors and is significant in determining the braid inductance. The average height is approximated by equation 11, where $b[\mathrm{~m}]$ is the gap between two adjacent spindles and is defined by equation 12 .

$$
\begin{gathered}
\hat{h}=\frac{2 d}{1+b / d} \\
b=\frac{2 \pi D_{m}}{C} \cos (\alpha)-N d
\end{gathered}
$$

It is recommended in [7] to compute the braid inductance term using the equation (13) where the term $\mathrm{S}$ (equation 14) is a more accurate estimation of the surface between the crossing conductors.

$$
\begin{gathered}
M_{b}=-\mu \frac{S}{N d} \frac{1-\tan ^{2}(\alpha)}{4 \pi D_{m}} \sin (2 \alpha) \\
S=N d h+b d \frac{\left(1-h^{2} / d^{2}\right)}{2}+N d^{2}\left(1-\frac{\pi}{4}\right)
\end{gathered}
$$

Kley reviewed analytical solutions in [5] and said that the height of the walls around the holes have to be considered for the magnetic field penetrating the hole produces Eddy current on the walls. This Eddy current creates a magnetic field which opposes to the one penetrating the hole. Hence, he introduced a semi-empirical corrective exponential term that reduces the value of the inductive term of $\mathrm{Z}_{\mathrm{t}}$. He also added a corrective factor to the definition of the transfer inductance $\mathrm{L}_{\mathrm{t}}$ to consider the curvature of the braid. For the sake of brevity, we directly used the complete definition given by [7] , which gave the most recent and most developed review on analytical models for transfer impedance determination.

\section{NUMERICAL STUDIES OF BRAIDED SHIELDS}

Numerical electromagnetic models has been less developed by authors because the necessary computing power is not available for long. The paper [14] introduces a numerical electromagnetic model of the braided shield, in order to obtain the transfer impedance. The 3D model, as show in figure 4 , respects the woven structure of the braid, which is hard to design. Though this model is very close to reality, the results of the transfer impedance processing was still a little different from the theory and measurement. The authors said that the discrepancies might come from the contact resistance between the strands, which is sensitive to small changes in the geometry. Using numerical electromagnetic model is considered as a good way to analyse and validate theorical approaches. Another paper from the same authors [15] introduced a simplified numerical model based on perforated tube and brought the same conclusion.

As far as we know, [14] gave the most reliable numerical model to compute the transfer impedance of a coaxial cable with braided shield. Hence, we use here the same sample of cable in order to obtain comparable results.

\section{PEERING NUMERICAL MODELING WITH THEORY}

\section{A. Simplified numerical model of the coaxial cable}

The model of coaxial cable shield we built was different of the one of [14] (figure4). We didn't use woven elementary conductors for the shield, as shown in figure 5. The features of the model are given in table I.

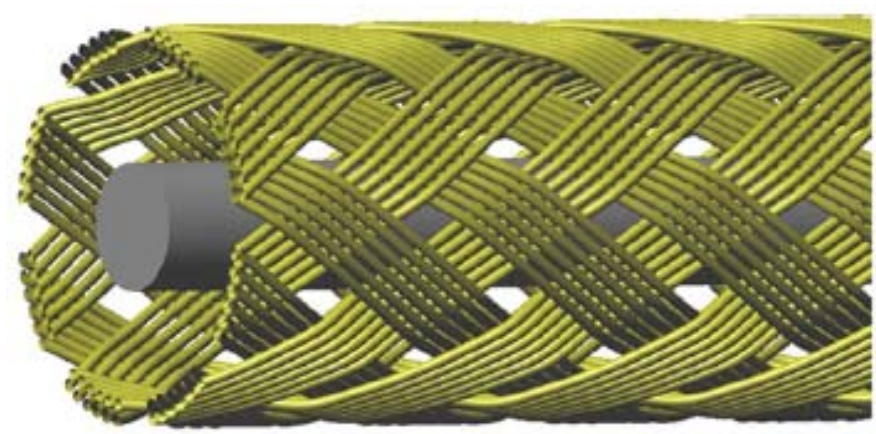

Fig. 4. 3D model of the braided coaxial cable shield from [14]

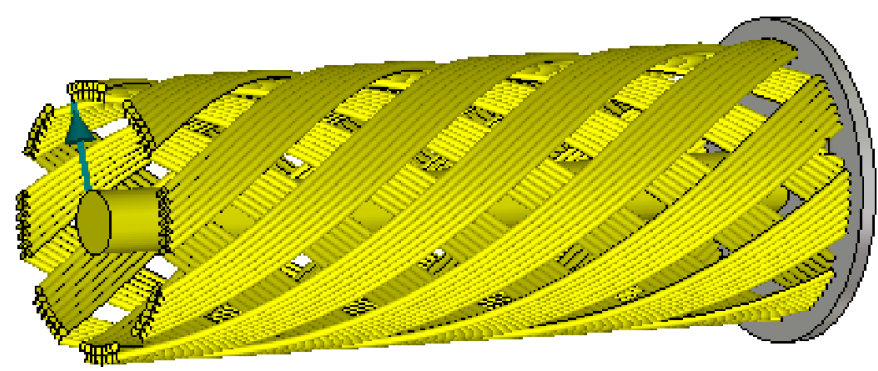

Fig. 5. Our simplified 3D model with nonwovens braid strands using CST microwaves 
TABLE I

BUILDING PARAMETERS OF RG58 COAXIAL CABLE FROM [15] AND DEDUCED SECONDARY PARAMETERS

\begin{tabular}{|c|c|}
\hline parameter & Value \\
\hline central conductor diameter $\mathrm{D}_{\mathrm{c}}$ & $0.8 \mathrm{~mm}$ \\
\hline Number of strands per spindle $\mathrm{n}$ & 7 \\
\hline Number of spindles $\zeta$ & 16 \\
\hline Strand diameter d & $120 \mu \mathrm{m}$ \\
\hline Internal shield diameter D & $3.02 \mathrm{~mm}$ \\
\hline Braid's metal & pure copper \\
\hline dielectric material & solid polyethylene \\
\hline Braid angle $\alpha$ & $32.32^{\circ}$ \\
\hline number of holes per unit length $\nu$ & $8629 \mathrm{~m}^{-1}$ \\
\hline Hole's magnetic polarizability $\mathrm{m}$ & 0.28 \\
\hline Hole inductance $M_{12}$ & $850 \mu \mathrm{H} . \mathrm{hole}^{-1}$ \\
\hline Braid inductance $M_{b}$ & $34 \mathrm{nH} . \mathrm{m}^{-1}$ \\
\hline filling factor $\mathrm{F}$ & 0.38 \\
\hline
\end{tabular}

The sample of cable is instrumented according to the figure 6 , which is a numerical implementation of the triaxial measurement setup presented in figure 1 and equation 1 is applied by using a post-processing. The results obtained for the simulation of the model of the figure 5 with the time domain solver of CST are shown in figure 7.

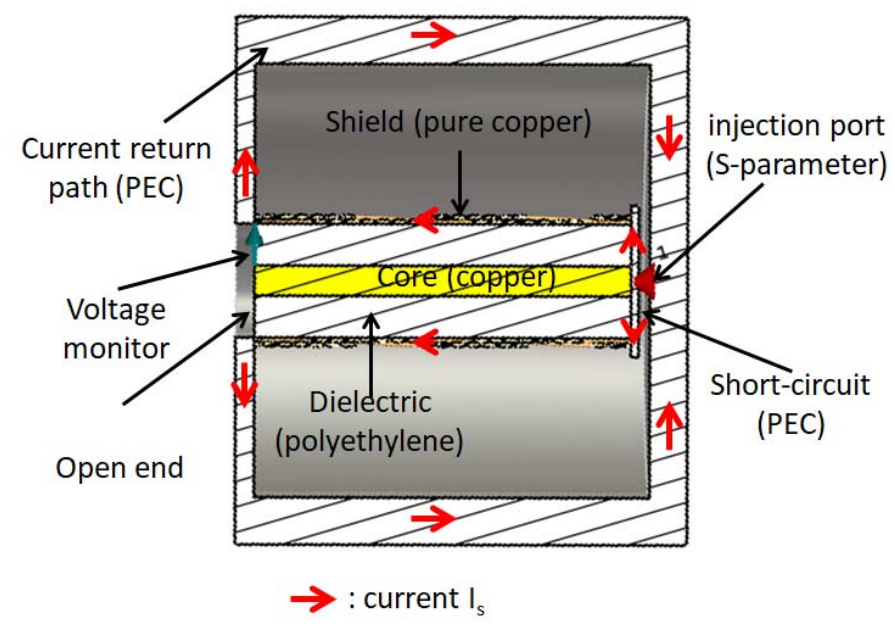

Fig. 6. Numerical implementation of the triaxial measurement setup to determine the transfer impedance $Z_{t}$

\section{B. correction of numerical results}

In [16], the authors reviewed the origins of the inductance term of $\mathrm{Z}_{\mathrm{t}}$. The $\mathrm{M}_{12}$ term from equation 5, now called the hole inductance $M_{h}$, can be completed by $M_{b}$ and $M_{s}$, namely the braid inductance and the skin inductance. The braid inductance $\mathrm{M}_{\mathrm{b}}$ arises from the woven structure of the braid and corresponds to the lack of our nonwoven model of coaxial braided shield. The skin inductance $\mathrm{M}_{\mathrm{s}}$ is linked to the eddy current circulating in the wall of the apertures when the magnetic field penetrates the holes. $\mathrm{M}_{\mathrm{s}}$ has been introduced in the semi-empirical formula revelopped by Kley in [5] and is not developed here for the sake of brevity. In order to correct the simulation result obtain with the simplified shield structure, we applied the equation 15 to the transfer impedance obtained with the numerical simulation.

$$
Z_{t, \text { corr }}=\left|Z_{t, \text { sim }}+j \omega M_{b}\right|
$$

Where $M_{b}$ is calculated using equations 13,14 and 11 .

\section{RESULT AND CONCLUSION}

The results corrected using $\mathrm{Z}_{\mathrm{t}, \mathrm{corr}}$ are compared in figure 7 with raw simulation result and measured data extracted from [17]. The frequency range of the extracted measurement data is limited to $[10 \mathrm{kHz} ; 1 \mathrm{GHz}]$, while the limitation of the simulated frequency range can be chosen much wider.

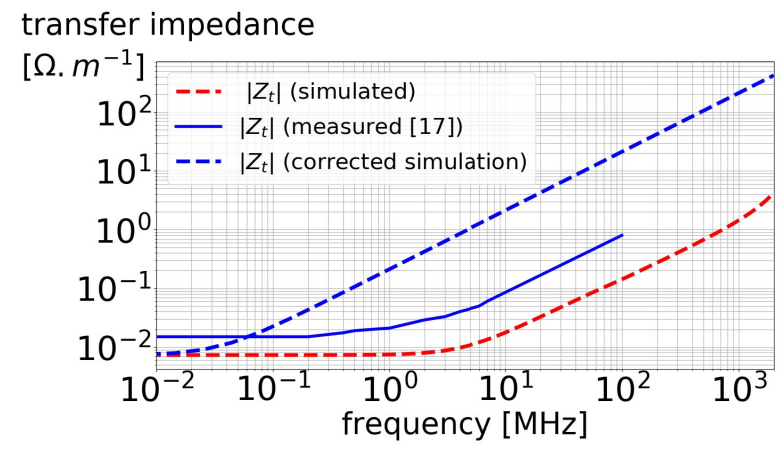

Fig. 7. Comparison between the measurement, raw results of simulation and corrected results of simulation of the magnitude of the transfer impedance $Z_{t}$ for a RG58 coaxial cable

\section{A. Results and interpretation and correction}

We see in the figure 7 that the raw result of the simulation is lower than the measured data extracted from [17]. This difference may merge from two sources. Firstly, the braid inductance term does not act in the simplified model. Secondly, the transfer impedance at low frequency, which is dominated by the DC resistance of the shield can change from one sample of RG58 to another. Though the RG58 model is standardized, cable manufacturers can use different material for the braid, for example pure copper or copper plated with tin or silver. The choice of the material can be a source of the discrepancy between the measurement and simulated data. The effect of the correction made by using the equation 15 obtain values of transfer impedance which are larger than the measured data from [17]. We considered that this was the effect of an overestimation of the transfer inductance $\mathrm{M}_{\mathrm{b}}$. We used a curve fitting between the results from simulation and measurement. We found a transfer inductance 26 time smaller than the one given by equations 13,14 and 11 and a DC resistance $7 \mathrm{~m} \Omega . m^{-1}$ higher. The results obtained with this second correction are shown in figure 8 .

We notice here that the curve corresponding the data obtained after applying the correction of the transfer inductance and add the DC resistance is much closer to the measured data. 


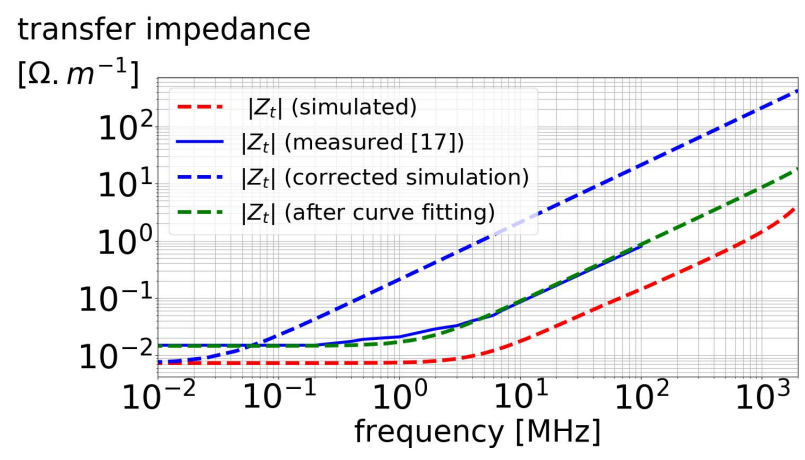

Fig. 8. Comparison between the measurement, corrected simulation before and after curve fitting of the magnitude of the transfer impedance $Z_{t}$ for a RG58 coaxial cable

\section{B. Conclusions}

In the paper, a novel approach to obtain the transfer impedance of coaxial cables with braided shield was considered. The transfer impedance was obtained using a combination of a simplified numerical electromagnetic model and analytic formulas. The model presented has an nonwoven structure as an approximation of the braided shield. The features of the model was corresponding to the standardized commercial coaxial cable RG58, the same as [17] considered, in order to obtain comparable results. The result of the simulation was corrected using an analytical formula from the review [7]. The formula used for the correction added a term corresponding to the effect of the weaving of the braid. The corrected result was higher than the measurement. We deduced that the braid inductance was overestimated in literature. We used a curve fitting in order to number the correction factor to apply. We found that the braid inductance had to be divided by 26 and the DC resistance incremented of $7 \mathrm{~m} \Omega . \mathrm{m}^{-1}$. The error on the braid inductance might come from the difficulty to calculate the average height of the carrier crossing. We thought error on the DC resistance might come from the choice of the material of the braid in our model, which can be different from the one of the cable used in the measurement taken as a comparison. Hence, we added $7 \mathrm{~m} \Omega \cdot \mathrm{m}^{-1}$ to the simulation results. The obtained data fitted better to the measurement data. The paper introduced a concept of simulation that can still be improved in order to assess the shielding properties of a coaxial cable design. Further work may use a well defined sample of cable in order to remove the difference caused by the ignoring of the exact building parameters of the cable used for measurement and comparison. Furthermore, we proposed to revalue the analytical formula determining the so called braid inductance by dividing the previous equation by 26. This correction factor may be reevaluated with our own measurement data.

\section{REFERENCES}

[1] S. A. Schelkunoff. The Electromagnetic Theory of Coaxial Transmission Lines and Cylindrical Shields. Bell System Technical Journal, 13(4):532-579, October 1934
[2] E. F. Vance. Shielding Effectiveness of Braided-Wire Shields. IEEE Transactions on Electromagnetic Compatibility, EMC-17(2):71-77, May 1975.

[3] Heinrich Kaden. Wirbelströme und Schirmung in der Nachrichtentechnik. Springer-Verlag, 1959. Google-Books-ID: WfFdDwAAQBAJ.

[4] Nathan Marcuvitz. Waveguide Handbook. IET, 1951. Google-Books-ID: Ao34iFuNZgIC.

[5] T. Kley. Optimized single-braided cable shields. IEEE Transactions on Electromagnetic Compatibility, 35(1):1-9, February 1993.

[6] S. Sali. An improved model for the transfer impedance calculations of braided coaxial cables. IEEE Transactions on Electromagnetic Compatibility, 33(2):139-143, May 1991.

[7] J. Verpoorte, H. Schippers, and J. H. G. J. L. Rotgerink. Advanced models for the transfer impedance of metal braids in cable harnesses. In 2018 IEEE International Symposium on Electromagnetic Compatibility and 2018 IEEE Asia-Pacific Symposium on Electromagnetic Compatibility (EMC/APEMC), pages 187-192, May 2018.

[8] David W. P. Thomas. Simulation of cable coupling Final Report, January 2009.

[9] E. F. Vance. Shielding effectiveness of braided wire shields, interraction note. April 1974.

[10] R. De Smedt and J. Van Bladel. Magnetic polarizability of some small apertures. IEEE Transactions on Antennas and Propagation, 28(5):703707, September 1980.

[11] F. De Meulenaere and J. Van Bladel. Polarizability of some small apertures. IEEE Journals \& Magazine, 1977.

[12] H. Schippers and J. Verpoorte. Hole inductance in braided cable shields. In 2015 IEEE International Symposium on Electromagnetic Compatibility (EMC), pages 56-61, August 2015.

[13] M. Tyni. The Transfer Impedance of Coaxial Cables with Braided Outer Conductor. pages 410-419, Wroclaw, Poland, 1975. Ser. Konfi.

[14] Ruben Otin, Jaco Verpoorte, Harmen Schippers, and Roger Isanta. A finite element tool for the electromagnetic analysis of braided cable shields. Computer Physics Communications, 191:209-220, June 2015.

[15] Ruben Otin, Jaco Verpoorte, and Harmen Schippers. Finite element model for the computation of the transfer impedance of cable shields. IEEE Transactions On Electromagnetic Compatibility, 53(4):950-958, 2011.

[16] H. Schippers, J. Verpoorte, and R. Otin. Electromagnetic analysis of metal braids. In 10th International Symposium on Electromagnetic Compatibility, pages 543-548, York, UK, September 2011.

[17] Michel MARDIGUIAN. Simple Method for Predicting a Cable Shielding Factor, Based on Transfer Impedance. emc Directory \& design guide:5, 2012. 